\title{
eJRIEPS
}

Ejournal de la recherche sur l'intervention en éducation physique et sport

11 | 2007

Varia

\section{Vers un cadre d'analyse pour différencier les modalités de « contextualisation authentique » en éducation physique et sportive}

Benoit Vercruysse, Ghislain Carlier et Léopold Paquay

\section{(2) OpenEdition}

\section{Édition électronique}

URL : https://journals.openedition.org/ejrieps/6945

DOI : $10.4000 /$ ejrieps.6945

ISSN : 2105-0821

Éditeur

ELLIADD

\section{Édition imprimée}

Pagination : 141-156

\section{Référence électronique}

Benoit Vercruysse, Ghislain Carlier et Léopold Paquay, « Vers un cadre d'analyse pour différencier les modalités de "contextualisation authentique » en éducation physique et sportive », eJRIEPS [En ligne], 11 | 2007, mis en ligne le 01 janvier 2007, consulté le 31 octobre 2021. URL : http:// journals.openedition.org/ejrieps/6945; DOI : https://doi.org/10.4000/ejrieps.6945

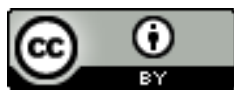

La revue eJRIEPS est mise à disposition selon les termes de la Creative Commons Attribution 4.0 International License. 
Vers un cadre d'analyse pour différencier les modalités de « contextualisation authentique » en éducation physique et sportive

Benoit Vercruysse*, Ghislain Carlier* \& Léopold Paquay**

Université catholique de Louvain, Louvain-la-Neuve, Belgique

* Unité d'éducation par le mouvement (EDPM)

** Groupe de recherche interdisciplinaire en formation des enseignants et en didactique (GRIFED)

\section{Résumé}

Comment aider l'élève à acquérir des connaissances, des savoir-faire et à l'amener à devenir compétent en situation? Quelles pratiques enseignantes effectives tournées vers la construction de compétences, pour quels effets? Pour les chercheurs qui tentent d'approcher et de comprendre les effets de la pédagogie par compétence, ces questions sont au cœur des débats.

Les théories se réclamant de la perspective située mettent en évidence l'intérêt d'étudier les relations entre le contexte et les apprentissages afin de mieux comprendre les situations scolaires. Au travers d'une revue de la littérature et d'illustrations tirées d'explorations, le présent article présente nos réflexions actuelles autour de l'observation de pratiques en se focalisant sur le concept de " contextualisation authentique ».

L'étude en cours a comme objectif d'identifier des ensembles de caractéristiques des pratiques enseignantes susceptibles d'influencer positivement le développement de compétences chez les élèves. Ce type de recherche devient une nécessité urgente pour la plupart des pays européens (Eurydice, 2002) ainsi qu'en communauté française de Belgique, où les dernières instructions officielles préconisent des démarches qui apprennent aux élèves à développer des compétences, c'est-à-dire à les rendre aptes à faire face à des situations complexes, significatives et " authentiques » en mobilisant des connaissances diverses (Beckers, 2002; Paquay, Carlier, Collès \& Huynen, 2001 ; Perrenoud, 1997; Rey, 2003; Roegiers, 2000). Afin de favoriser un apprentissage mobilisable, transférable à d'autres situations, certains auteurs proposent de les contextualiser de manière plus « authentique " en rapport à la vie extrascolaire. De nombreux travaux en psychologie de l'éducation (Pintrich \& Shunck, 1996 ; Pintrich, 2003) 
montrent que la variable contextualisation constitue une des conditions fondamentales d'un apprentissage en profondeur.

Le présent document a comme objectif d'exposer la construction d'un modèle autour de la notion de «contextualisation authentique » comprenant différentes dimensions des situations d'enseignement-apprentissage. II est illustré par une des recherches exploratoires en abordant son recueil de données ainsi que l'analyse des caractéristiques de la pratique enseignante.

\section{La contextualisation d'une situation d'enseignement-apprentissage}

Comme le souligne Tardif (1999, p. 15): «Dans les sociétés, les institutions scolaires doivent faire en sorte que les connaissances construites et les compétences développées par les élèves soient caractérisées par le plus haut degré de transférabilité possible. Si ces connaissances ne sont pas transférables en dehors des murs de la classe, cela signifie que toutes les années passées à l'école n'ont servi qu'à susciter des apprentissages scolaires ». Selon lui, le rôle de l'enseignant serait de mettre en place des situations qui sont susceptibles de favoriser des compétences utiles à la vie en dehors de l'école. II ajoute aussi que ces compétences doivent permettre de servir à la réflexion, à la compréhension et à l'action par rapport aux phénomènes de la « vraie » vie.

De nombreux auteurs issus de la perspective située, tant psychologues qu'anthropologues, ont développé des cadres théoriques investiguant notamment les relations indissociables entre contexte et apprentissage, d'une part et entre contexte et cognition, d'autre part. Ils considèrent qu'apprendre, c'est participer à des pratiques d'une communauté socioculturelle. Ayant étudié des communautés traditionnelles, ils ont observé que les apprentis tailleurs directement baignés dans le contexte professionnel s'engagent plus volontiers dans la tâche (Lave \& Wenger, 1991). L'analyse des situations de classe doit être distinguée de situations traditionnelles pour plusieurs raisons qu'il est nécessaire de détailler.

Mottier Lopez (2005) a étudié les différences entre les contextes scolaires et les contextes dits d' " apprentissages traditionnels ». Elle fait remarquer que chaque contexte possède ses caractéristiques propres mais également qu'il a sa propre nature de savoirs. Dans les communautés traditionnelles, les savoir et savoir-faire sont spécifiques aux gestes du métier de l'expert, alors que dans le contexte scolaire, l'objectif est de s'approprier des savoir-faire généraux utiles pour n'importe quel métier et pour la vie en société. La nature du savoir est également différente et les productions sont porteuses de significations différentes. Lave et Wenger (1991) montrent que dans les communautés dites 
« traditionnelles », le processus identitaire est spécifique et proximal (l'apprenti se prépare à accéder au métier du maître qu'il côtoie). En revanche, l'étude de Allal et Mottier Lopez en 2004 montre que chez des enfants de l'école primaire en classe de mathématiques, le processus identitaire est diffus et découplé dans le temps (l'élève se prépare à partager la culture générale véhiculée par l'enseignant; son identité socioprofessionnelle se définira plus tard).

Cette vision novatrice des communautés de classe permet d'analyser les situations quotidiennes comme des pratiques sociales, dans la mesure où elles s'inscrivent et prennent forme dans des systèmes d'activités qui véhiculent des processus sociaux, des normes et des valeurs (Lave, 1992 in Mottier Lopez, 2003). Selon cette conception, transposée au milieu scolaire, l'élève apprend lorsqu'il s'approprie activement la culture de la classe et les outils intellectuels avec lesquels il va pouvoir agir en situation. C'est au travers d'un fonctionnement en communauté qu'il va apprendre à agir. Les situations de classes seraient vues comme des espaces d'échanges de connaissances, de cultures, de valeurs, de normes qui au fil de l'évolution du contexte donneraient du sens aux apprentissages. L'école n'est plus vue comme un milieu d'apprentissage déconnecté de la « vraie » vie mais comme un « vrai » milieu, en ce sens qu'il est considéré comme représentatif de la société ayant sa culture, ses normes, ses valeurs.

Deux principes à la base de la conception contextualiste du transfert des apprentissages retiennent particulièrement notre attention.

Le premier postule que le traitement de l'information est indissociable du contexte dans lequel il prend place (Greeno, 1994 in Mottier Lopez, 2005). Et le second postule que ce contexte d'enseignement et d'apprentissage influence de façon déterminante la représentation et l'utilisation qui sera faite des connaissances (Frenay \& Bédard, 2004). Et donc, si l'on veut s'intéresser à la manière dont les connaissances seront réutilisées et à la manière dont l'élève sera engagé, apparaît la nécessité d'étudier ce contexte d'apprentissage et de vérifier dans quelle mesure il est en relation avec les apprentissages et avec l'engagement dans la tâche. De plus, le rapport aux apprentissages doit être le plus similaire possible dans la situation d'apprentissage et dans la situation d'utilisation. C'est ce qu'indique Mottier Lopez. En décrivant les processus participatifs de la microculture de classe, elle recommande la promotion de situations permettant un engagement significatif de l'apprenant dans la situation scolaire tel qu'il serait dans des situations quotidiennes extrascolaires.

Dans la littérature anglo-saxonne, les auteurs parlent d'authenticité des situations d'enseignement/apprentissage, en rapport avec les situations de mobilisation des acquis 
(ex. : Elliot \& Dweck, 2005). Cette notion émane notamment de chercheurs qui considèrent que les pratiques scolaires, lorsqu'elles ne sont pas reliées à celles du monde extrascolaire, favorisent l'acquisition de connaissances "inertes » que les élèves ne parviennent pas à remobiliser dans d'autres lieux d'activités sociales. Convaincus que les cognitions construites par l'apprenant sont fondamentalement liées au contexte dans lequel elles ont été acquises, ces chercheurs préconisent de promouvoir en classe des situations les plus proches possibles des conditions de la vie extrascolaire (cf. les courants « situated cognition/learning »: Brown, Collins \& Duguid, 1989 ; Lave \& Wenger, 1991 ; Bédard, Frenay, Turgeon \& Paquay, 2000). Selon Brown et al. (1989) les pratiques authentiques sont «les pratiques ordinaires de la culture », vues comme des pratiques signifiantes, finalisées et cohérentes dans le groupe socioculturel qui les produits.

Frenay et Bédard (2004) font la proposition de situations d'Apprentissage-Enseignement Contextualisé authentique (AECA), par rapport à deux principes : l'authenticité du contexte et le compagnonnage cognitif. Ils ont dégagé sept indicateurs de ce qu'ils nomment l'« authenticité du contexte de formation », adaptée à l'enseignement supérieur professionnalisant : le respect du contexte de mobilisation ; la visée de développement de compétences ; des situations complètes et complexes ; des contenus pluridisciplinaires ; des situations-problèmes multidimensionnelles, diversifiées, ouvertes sur des solutions / conclusions / interprétations multiples. Bédard (à paraître) pose que chacun de ces indicateurs peut être présent à différents degrés (faible, moyen ou élevé). Ainsi, par exemple, selon Frenay, Bédard, Turgeon et Paquay (2000), il existe trois contextes de mobilisation qui permettraient de donner lieu à des transferts de natures différentes et permettant de motiver différemment les élèves : à l'intérieur d'un même cours, à l'intérieur d'un même programme de formation et lors d'activités hors programmes. Ce sont Collins, Brown et Holum (1991) qui élaborent le modèle du compagnonnage cognitif (cognitive apprenticeship) en prenant exemple sur l'apprenti dans des communautés de pratiques traditionnelles. Celui-ci apprend son métier « sur le tas », en regardant faire et en faisant, supervisé par un expert qui vise à le rendre progressivement autonome par quatre attitudes: la modélisation, l'étayage, le retrait graduel et le coaching. Trois différences caractérisent cet apprentissage : les stratégies de résolution de problèmes de l'expert sont visibles, les tâches sont situées dans un contexte de travail finalisé, les compétences et connaissances sont justifiées par la complexité de la tâche visée. Notre enjeu est donc de repérer ces dimensions dans l'enseignement général en Communauté française de Belgique. 
La revue de la littérature francophone et anglo-saxonne nous amène à adapter ces caractéristiques à l'enseignement secondaire supérieur. Entre autres, Roegiers (2000) propose de placer les élèves face à des situations qui font sens : elles doivent interpeller l'élève, lui poser un défi, lui paraître utiles, lui permettre de contextualiser les savoirs, l'amener à une réflexion épistémologique et mettre en évidence l'apport des autres disciplines dans la résolution de la tâche.

Le Boterf (1997, p. 54), en décrivant ce qu'est une compétence professionnelle, insiste sur l'aspect finalisé et situé de la compétence. "S'il n'y a de compétence que mise en acte, la compétence ne peut être que compétence en situation. Elle ne préexiste pas à l'événement ou à la situation. Elle s'exerce dans un contexte particulier. Elle est contingente. Il y a toujours " compétence de " ou " compétence pour ». Ce qui revient à dire que toute compétence est finalisée (ou fonctionnelle) et contextualisée. " De même, Tardif (1999) ajoute que pour qu'un apprentissage soit transférable, il est impératif qu'il soit finalisé, c'est-à-dire qu'il soit viable ou qu'il concoure à la compréhension de divers tâches cibles et à leurs réalisations.

La classification de Perkins (cité par Tardif, 1999), quant à elle, différencie deux types d'environnements pédagogiques. Les environnements minimalistes mettant l'accent sur la transmission des informations, sur le langage où il y a peu d'occasions et peu de moyens pour l'élève d'explorer des problématiques authentiques et de résoudre des problèmes complexes. Et les environnements maximalistes où la majorité du contrôle est laissé à l'élève et où il s'engage dans la réalisation d'activités qui poursuivent d'une façon concomitante plusieurs objectifs d'apprentissage (Tardif et Presseau, 1998). Perkins estime que la majorité des écoles actuelles ne proposent que des environnements minimalistes. Avec Tishman et Jay (1995 cité par Tardif, 1999), Perkins propose quatre orientations permettant de créer et de gérer pédagogiquement des environnements maximalistes : soulever des questions, fournir des arguments, apporter des justifications et adopter un point de vue critique. « Ces logiques mettent l'accent sur l'élève lui-même... La création d'environnements pédagogiques dans le respect de telles orientations fait en sorte que la compréhension de différents phénomènes et la possibilité d'agir sur les questions et les problématiques qu'ils posent en dehors des murs de l'école constituent fréquemment le déclencheur des apprentissages (Tardif, 1999, p. 172). Comme le souligne Tardif, dans ce type d'environnements, l'accent est mis sur la réalisation d'activités en relation avec le « dehors » de l'école par la gestion de plusieurs objectifs d'apprentissage de complexité adaptée au niveau des élèves. 
En synthétisant les apports de la littérature, on peut donc dire qu'une pratique enseignante est contextualisée de manière authentique lorsqu'elle fait référence à des situations externes à la classe et que les tâches demandées aux apprenants sont finalisées et complexes. Nous distinguons, au travers de la littérature existante, les tâches et ressources mises à disposition de l'élève, d'une part et l'intervention de l'enseignant, d'autre part. En effet, le modèle du compagnonnage cognitif décrit l'attitude de l'expert, ou dans notre cas l'enseignant, qui sert de guide plutôt que d'un modèle à atteindre, mais compétent pour accompagner l'élève dans son processus d'apprentissage.

Tableau I. Modalités de contextualisation authentique.

\begin{tabular}{|l|l|l|}
\hline & $\begin{array}{l}\text { Interventions de } \\
\text { l'enseignant } \\
(\mathrm{I})\end{array}$ & $\begin{array}{l}\text { Tâches demandées aux } \\
\text { élèves } \\
\text { et ressources mises à } \\
\text { disposition }(\mathrm{T})\end{array}$ \\
\hline Externalité $(\mathrm{E})$ & $\mathrm{El}$ & $\mathrm{ET}$ \\
\hline Finalisation $(\mathrm{F})$ & $\mathrm{Fl}$ & $\mathrm{FT}$ \\
\hline Complexité $(\mathrm{C})$ & $\mathrm{Cl}$ & $\mathrm{CT}$ \\
\hline
\end{tabular}

\section{Cadre d'analyse des formes de " contextualisation "}

Sur la base de ce qui précède, dans le cadre de notre recherche, nous retenons actuellement trois dimensions interreliées de la « contextualisation authentique »:

a. la référence externe à un ailleurs et/ou à un autre moment que ce qui relève de l'icimaintenant de la classe. Dans le cadre d'un cours, il est fait référence à des faits d'actualité et de société ; à l'actualité culturelle (artistique et sportive); à la vie professionnelle; au quotidien des élèves (vie affective, groupale, familiale) ; il est fait recours à des ressources extérieures (visites de professionnels à l'école ou rencontres sur leur lieu de travail) ;

b. la finalisation des activités demandées aux élèves et leur pouvoir mobilisateur supposé : confrontation à des défis, des situations-problèmes, des projets... ; c. le degré de complexité par rapport au niveau cognitif des apprenants: les activités nécessitent des opérations diverses ; elles sont ouvertes (plusieurs solutions possibles) ; elles nécessitent des ressources multiples...

Pour chacune de ces dimensions, nous avons déjà relevé quelques modalités et indicateurs concernant: les tâches assignées aux apprenants et les ressources mises à leur disposition, les interventions de l'enseignant. 
ET Les activités réalisées par les élèves et/ou les ressources mobilisées font référence à des phénomènes de société, ou à des fonctionnements de professionnels, ou à des vécus expérientiels des élèves (individuellement ou collectivement) ;

El L'enseignant affirme ou explicite le lien de similitude entre une activité en classe et des situations externes et/ou il donne des exemples ; il fonctionne lui-même comme un expert dans le domaine abordé (par exemple, s'il pratique la discipline sportive enseignée) ; il exploite l'expérience extrascolaire des élèves ;

FT Les tâches des élèves sont finalisées vers un défi, un problème à résoudre, un projet à réaliser ; ou elles s'inscrivent dans un projet global mobilisateur ; cette finalisation peut être intrinsèque et/ou extrinsèque ;

FI L'enseignant fait découvrir le but, le sens, l'utilité des activités (pour les autres branches, la vie professionnelle, personnelle, quotidienne); il démontre cette utilité ; il explique et/ou co-construit avec les élèves le sens que l'activité peut avoir pour eux (selon leurs intérêts, besoins, connaissances préalables) ;

$C T$ Les situations auxquelles les élèves sont confrontés sont « complexes »;

Cl L'enseignant accompagne la confrontation à la complexité : il modélise, étaye l'activité afin d'encourager les élèves; il désétaye progressivement (compagnonnage cognitif).

Les apprentissages en classe se définissent au travers d'espaces d'échanges, de connaissances, de cultures, de valeurs qui, au fil de l'évolution du contexte, donnent sens aux apprentissages. Dès lors, notre intérêt pour une description la plus complète des situations de classe nous oblige à intégrer un ensemble de caractéristiques qui permettent de décrire les activités spontanées des élèves. Lors des observations exploratoires, nous avons observé des élèves qui, en cours d'activité, ont amené spontanément leur culture dans l'espace d'interaction. Nous en avons vu au cours de Basket-ball amener la culture « streetball » en réalisant des mouvements spécifiques et des attitudes codifiées. En gymnastique sportive, en réalisant des prouesses techniques inspirées du «breakdance ». Nous constatons que les élèves apportent eux aussi leur culture sportive au sein de la classe et ce parfois dans une direction non planifiée par l'enseignant. Ces constats nous forcent à intégrer de tels comportements qui rendent compte du contexte effectif de la classe. L'analyse future consistera à voir dans quelle mesure il y a réellement coconstruction de savoir et de savoir-faire ou si l'on parle de co-existence de cultures différentes. Dès lors, la distinction activités demandées par l'enseignant/activités réalisées s'ajoute au modèle. 
Tableau II.Modalités de contextualisation authentique (2).

\begin{tabular}{|l|l|l|l|}
\hline & $\begin{array}{l}\text { Interventions } \\
\text { l'enseignant } \\
(\mathrm{I})\end{array}$ & $\begin{array}{l}\text { Tâches demandées } \\
\text { aux élèves } \\
\text { et ressources mises àches réalisées par } \\
\text { disposition (TD) }\end{array}$ & $\begin{array}{l}\text { Tâces élèves } \\
\text { et ressources mises à } \\
\text { disposition (TR) }\end{array}$ \\
\hline $\begin{array}{l}\text { Externalité } \\
(\mathrm{E})\end{array}$ & El & ETD & ETR \\
\hline $\begin{array}{l}\text { Finalisation } \\
(\mathrm{F})\end{array}$ & $\mathrm{Fl}$ & FTD & FTR \\
\hline $\begin{array}{l}\text { Complexité } \\
(\mathrm{C})\end{array}$ & $\mathrm{Cl}$ & CTD & CTR \\
\hline
\end{tabular}

\section{Etude exploratoire}

Cette étude se présente en deux phases. La première est constituée d'une analyse de séquences visant à dégager des caractéristiques de pratiques enseignantes dans des séquences observées chez neuf enseignants expérimentés en éducation physique (Vercruysse, 2005). La seconde rapporte des observations filmées chez quatre enseignants expérimentés et vise à questionner les catégories pré-établies de la « contextualisation authentique ». Dans le présent article, la démarche méthodologique ainsi que le déroulement de la première phase seront abordées. Ensuite, une illustration plus détaillée des catégories du concept de «contextualisation authentique » sera présentée. II est nécessaire, afin d'en mesurer l'implication, de situer la démarche méthodologique.

3. 1. Analyse de la pratique enseignante sous l'angle de la pédagogie par compétence

Les observations ont été menées chez neuf enseignants en éducation physique dont six hommes $(\mathrm{H} 1-\mathrm{H} 6)$ et trois femmes (F1-F3) (voir tableau 3 ci-dessous). Grâce à des observations directes, à des prises de notes dans un journal de bord et à des entretiens post-séance, notre objectif a été de prendre un maximum d'informations de nature différentes en laissant s'exprimer l'« acteur » dans son gymnase, terme utilisé par Léziard (2004, p. 15) pour désigner la personne agissant et produisant de l'enseignement et susceptible de verbaliser ses actions.

Trois types de données émanent du corpus. Les données recueillies par observation directe (O), constituées des caractéristiques des tâches proposées (description, objectif, lancement de la tâche, réalisation effective...), de l'intervention verbale et 
comportementale du professeur (feedback, relance, régulations, aide) et des attitudes d'élèves. Ensuite, des données ont été récoltées lors de l'entretien post-séance avec la technique dite des Instructions au sosie (I) qui privilégie le discours propre comme moyen de cerner la pratique professionnelle. Dans le travail de Oddone (1981), le travail d'instructions au sosie portait sur quatre domaines de l'expertise professionnelle. Les rapports aux pairs dans le(s) collectif(s), les rapports à la hiérarchie, les rapports aux organisations formelles ou informelles du monde du travail, et enfin le dernier particulièrement saillant dans le cadre de notre recherche : les rapports à la tâche. Ceux-ci sont pour nous prioritaires par le fait même qu'ils questionnent l'action didactique en regard de l'appropriation personnelle de l'activité enseignante tourné vers la construction de compétences. Comme le souligne Sauvegrain et Terrisse (2003), apparaît aujourd'hui la nécessité d'interroger le rapport singulier et personnel qu'entretient le sujet à l'activité d'enseignement. Nous nous intéressons donc dans une première partie de la recherche, aux singularités de l'objet en y intégrant le point de vue du sujet afin de comprendre les différences entre travail prescrit et travail réel. La technique de l'instruction au sosie vise à "donner au sujet l'espace d'élaboration et l'occasion de découvrir le réel de son activité » (Clot, 1999, p.148). Du point de vue du chercheur, cette technique caractérisée par un scénario typique, facilite l'entrée dans l'interview.

Nous rejoignons Amigues (1994, p.53) et son souci d'appréhender l'activité du professeur à l'intérieur d'un "contexte de pratiques, dans lequel les actions des protagonistes sont culturellement situées et distribuées". Cela nous a conduit à nous inscrire à cette phase de la recherche dans une approche à la fois ergonomique et située. Notre regard tourné vers le professeur tente d'élaborer une culture chez le chercheur et est issu de la confrontation des expériences singulières. Les singularités et régularités auxquelles notre analyse donne lieu, tout comme l'appropriation personnelle de cette culture par le chercheur nous aident à réinscrire les différents vécus. Enfin, viennent les données récoltées par une corroboration (Co) sur des interprétations directement présentées par le chercheur. Afin d'analyser les différentes pratiques, nous avons utilisé l'outil générique Comp.A.S. ${ }^{1}$ qui permet d'analyser des situations de formation dans l'enseignement obligatoire, dans l'enseignement supérieur et dans les formations professionnelles. Il a été développé à l'origine avec des enseignants et des formateurs d'enseignants (Parmentier \& Paquay,

\footnotetext{
${ }^{1}$ Cet outil Compas reste ouvert et est toujours en développement. II peut être appliqué pour analyser des situations diverses d'enseignement/apprentissage et est téléchargeable sur le site < www.grifed.ucl.ac.be >.
} 
2002) et il présente un modèle organisant différentes facettes de l'enseignement susceptibles de favoriser le développement de compétences. Dans la présente recherche, notre point d'attention se situe, en lien avec la "contextualisation » des situations d'enseignement et d'apprentissage, autour de la dimension «L'enseignant organise des situations problèmes " de l'outil. Cette dimension contient plusieurs indicateurs (1.1, 1.2, 1.3, 1.4 dans le tableau 1) et tente de rendre compte l'ensemble des éléments.

II nous a semblé nécessaire - dans une recherche exploratoire de nature qualitative - de nous focaliser sur cette dimension apparaissant comme "marquantes" pour cet enseignant. Pour ce faire, une analyse a été réalisée sur ce partenaire ayant des indicateurs qui présentaient au moins deux des trois caractéristiques $(\mathrm{O})$, (I) et (Co) et qui totalisaient le nombre le plus important d'indicateurs par dimension. (Voir lettres grisées dans le tableau III)

Tableau III. Comp.A.S.

\begin{tabular}{|c|c|c|c|c|c|c|c|c|c|}
\hline & $\mathrm{H} 1$ & $\mathrm{H} 2$ & $\mathrm{H} 3$ & $\mathrm{H} 4$ & $\mathrm{H} 5$ & $\mathrm{H} 6$ & F1 & F2 & F3 \\
\hline $\begin{array}{l}\text { 1. L'enseignant organise } \\
\text { des situations problèmes... }\end{array}$ & & & & & & & & & \\
\hline $\begin{array}{l}1.1 \text { II confronte l'apprenant à } \\
\text { des situations complexes qui } \\
\text { sont contextualisées ou } \\
\text { proches de situations de vie. }\end{array}$ & & I & & & $\begin{array}{l}\mathrm{O} \\
\mathrm{Co}\end{array}$ & & I & & \\
\hline $\begin{array}{l}1.2 \text { L'enseignement est } \\
\text { organisé autour de cas, de } \\
\text { situations et de tâches } \\
\text { motivantes et susceptibles de } \\
\text { constituer un défi qui prend } \\
\text { sens pour l'apprenant. }\end{array}$ & | & & $\begin{array}{l}0 \\
1\end{array}$ & $\begin{array}{l}0 \\
1\end{array}$ & $\begin{array}{l}0 \\
1 \\
\text { Co }\end{array}$ & & & I & \\
\hline $\begin{array}{l}1.3 \text { II met en place des } \\
\text { démarches de pédagogie par } \\
\text { projets. }\end{array}$ & & & & & I & & & I & I \\
\hline $\begin{array}{l}1.4 \text { II met en place des outils } \\
\text { et des conditions pour que } \\
\text { l'apprenant fasse une } \\
\text { démarche active de } \\
\text { résolution de problèmes. }\end{array}$ & & & & & $\begin{array}{l}\mathrm{O} \\
\mathrm{Co}\end{array}$ & & & & \\
\hline
\end{tabular}


Le choix du contenu de chaque facette a donc été doublement déterminé par la qualité des données (verbatims d'enseignants, observations) et par l'occurrence des différents indicateurs au sein même d'une même "facette" d'enseignement. Dans la continuité de la démarche, une analyse qualitative approfondie des différents types de données en relation avec les catégories de la "contextualisation» a été intégrée pour comprendre les conditions de réalisation dans le contexte de la classe.

3. 2. Illustration des catégories de la " contextualisation authentique "

Le premier exemple nous montre en quoi, dans l'espace entre les actions donc dans l'inter-action, l'enseignant tente de donner du sens aux apprentissages. Lors d'un entretien, il nous dit : "Par exemple, on fait de l'endurance pour l'instant, un éducateur est passé dans la cour et je savais qu'il courait bien... je l'ai interpellé. "Tu ne veux pas dire ce que tu fais comme activité sportive et à quel rythme ?". Et puis là, il était tout content et il a commencé à raconter sa pratique personnelle. "Ah oui, j'ai fais le tour du Mont Blanc sur $150 \mathrm{~km}$ en $40 \mathrm{~h}$ avec des dénivelés positifs et négatifs de $8000 \mathrm{~m}$. Et les élèves ont commencé à poser des questions: C'est où le Mont Blanc Monsieur? "Tu pourrais expliquer longtemps l'entraînement des triathlètes, mais si tu leur montres quelqu'un qui a pratiqué, là, la théorie devient du concret. Et au moins, ils ont compris. Et j'ai parlé de l'âge en disant que la personne était au départ dynamique et tonique et a transféré au fil des années son exercice en course d'endurance. Il continue son activité mais ne sait plus être aussi dynamique qu'un jeune de 20 ans. C'est pourquoi, l'on voit des gens de 40 ans qui font de l'endurance. »

II nous dit par la suite: "Les élèves s'en souviennent plus que d'un discours, ils ont $d u$ concret ». II explique en même temps qu'il a un fil conducteur dans son cours et que ces exemples viennent instinctivement.

Si ce n'est un aspect de l'expertise de l'enseignant tel la capacité instantanée à profiter de la situation, cette caractéristique pourrait s'inscrire dans notre modèle. Dans cet exemple$\mathrm{ci}$, on peut se trouver dans la combinaison ETD et El qui définit le degré d'externalité de l'activité (ici en situation d'explication de savoirs et de savoir-faire) ainsi que la caractéristique du discours de l'enseignant expliquant le lien de similitude entre les tâches demandées aux élèves et d'hypothétiques situations futures. Le contexte a un degré d'externalité (présence de l'éducateur témoin d'une pratique de référence) ainsi que l'intervention de l'enseignant expliquant le lien de similitude entre l'endurance à l'école et le monde extrascolaire. 
Le second exemple du corpus illustre d'autres formes de contextualisation authentique. Un partenaire nous dit lors de la corroboration : "Ce qui m'intéresse de plus en plus en " audace en sécurité ${ }^{2}$ ", c'est ce que j'ai développé au niveau collectif et handicap. C'est tout un "bazar " avec l'idée d'audace en sécurité collective. Par exemple : on est dans un lieu qui prend feu et on doit évacuer. Je mets la situation tellement difficile que c'est irréalisable seul. On ne peut réussir que si l'on est aidé. Mais avant, on apprend aussi lors d'une séance à maîtriser les chutes. Il faut le faire. Chutes avant, latérales, arrière, des croche-pieds... Par exemple, on a un mur énorme à franchir constitué d'un mousse énorme entre deux barres parallèles. Ils n'ont pas droit aux montants verticaux mais on a droit aux barres et au mousse. Dans chaque groupe de cinq, je sais qu'il y a un ou deux qui n'y arriveront pas seul. Ils doivent trouver une stratégie de groupe et c'est chronométré. Le but est de sortir tout le monde le plus vite possible. Mes situations sont déjà fort attractives, et à certains moments il y a des challenges. Il y a des moments avec le chrono et des moments avec de la qualité... Tu sais, j'amène une sirène avec un girophare dans la salle. Un tapis, un Sarneige. Ils sont six ou sept dessus. On imagine une salle de danse, une discothèque. Le tapis, c'est le départ de la situation. Et à la fin, ils doivent tous arriver sur un plinth et c'est la situation finale. C'est la sortie. Et donc jusqu'à l'arrivée, c'est le chrono qui tourne.

Ils ont une séance où c'est de l'entraînement comme ils veulent. Ils ont le parcours à évacuer et ils le prennent où ils veulent mais ils s'entraînent obligatoirement en équipe. Puis un premier chrono: on regarde ce qui va. Et ce qui est intéressant, c'est qu'ils regardent les autres équipes. Ils se disent : "Ah, tu as vu ils font comme cela ». Et puis ils essayent. Et ce que je leur dit, mais pas au début, c'est que la seule chose qui m'importe aux deux chronos, c'est que leur équipe progresse par rapport à leur temps à eux. Ce n'est pas du tout une compétition entre les autres. Mais cela je ne leur dit pas au début, c'est important. Après je prends le même parcours mais je mets un aveugle. Dans le groupe, il y a un copain à eux qu'ils ont emmenés à la discothèque. J'invente un petit scénario: "On invite un copain aveugle à la discothèque, et mince! Il y a le feu. Cela commence, il faut sortir. Ah ! doucement on ne peut pas laisser notre copain. Là, ce n'est pas possible, donc on doit le sortir du feu. " Le même parcours mais avec un aveugle. Après le même parcours avec un handicapé et j'attache une jambe avec un bras et là, ils

\footnotetext{
${ }^{2}$ L'audace en sécurité est une activité qui permet à l'élève d'expérimenter des tâches variées en utilisant les coordinations de base dans des situations multiples de hauteur et de profondeur.
} 
doivent vraiment mettre en place une stratégie. On est en audace en sécurité mais collective. »

Cet extrait nous montre comment l'expertise de l'enseignant amène par un scénario contextualisé une véritable complexité finalisée en milieu scolaire. Plusieurs éléments émergent en rapport à la construction de notre modèle. Suivant notre classification entre tâche et intervention de l'enseignant, nous pouvons distinguer ce qui relève des caractéristiques des situations mises en place et l'intervention de l'enseignant lançant et régulant l'activité. Par exemple, la complexité de la tâche (CTD) se manifeste par le fait qu'il y a différentes difficultés à gérer en même temps (chronomètre qui tourne, techniques de franchissement d'obstacles, travail collectif...). L'accompagnement à la complexité (Cl) s'explique par l'aspect progressif des différentes tâches (entraînement aux chutes, entraînement sur le parcours en équipe, réflexions sur la base des prestations des autres...). De plus, suite à la modélisation de la situation, étaye par un enchaînement d'activités pour en arriver à un retrait progressif. II laisse la responsabilité aux équipes en tentant de se rapprocher de l'autonomie de gestion et de fonctionnement. La référence externe de cette tâche (ETD) se situerait, quant à elle, dans ce que l'on pourrait appeler l'habillage du lancement de la situation en créant un contexte susceptible de faire sens pour l'élève ; invention d'un scénario, matériel extérieur, sirène et girophare. Dans les faits, les élèves sont confrontés à une tâche en contexte scolaire (environnement matériel artificiel) mais possédant des caractéristiques proches du contexte d'une hypothétique situation-cible (chronomètre symbolisant l'aspect urgent du feu, en équipe avec handicap) et de plus susceptible de constituer un défi à résoudre par l'apprenant (FTD). Enfin, ne serait-on pas dans le cas d'un environnement maximaliste qui pourrait permettre notamment de déclencher des apprentissages?

\section{Conclusion}

A l'heure actuelle, les professionnels impliqués dans la formation ou dans l'éducation ne peuvent plus penser ni agir sans faire référence à la notion de compétence. Cette visée éducative tournée vers l'avenir est primordiale si l'on ne veut pas s'en tenir à faire développer chez les élèves des connaissances inertes seulement utiles à l'école. Le praticien détient peut-être une clef permettant de favoriser l'acquisition de connaissances mobilisables : la contextualisation authentique des savoirs.

L'enjeu de cette recherche est de comprendre les pratiques enseignantes sous cet angle. Qu'est-ce qui est authentique dans une situation de classe, à la fois dans ce que 
l'enseignant met en place et à la fois dans ce que fait et perçoit l'élève ? Plusieurs constatations et interrogations majeures sont nées suite à cette étude.

Sur la base des études exploratoires, l'essai de classification est, à notre sens, prometteur car il permet de différencier les aspects du contexte de formation et d'en classer les modalités par rapport au concept de contextualisation authentique que l'on tente d'adapter à l'enseignement général. Les explorations visant à rendre compte de telles pratiques sont néanmoins insuffisantes pour appréhender les processus de contextualisation décontextualisation - recontextualisation qui selon Tardif est le cadre de compréhension du transfert des apprentissages.

La question qui est posée suite à cette étude concerne les effets de telles pratiques. Si les situations mises en place par l'enseignant possèdent des caractéristiques susceptibles de favoriser le développement de compétences, elles favoriseraient donc le transfert des connaissances. L'élève, lui-même, perçoit-il le sens et l'utilité de ces différentes tâches ? Qu'en est-il dès lors des répercussions de ces perceptions sur la motivation à s'engager dans la tâche et sur la valeur que les élèves accordent à cette tâche ? Ces différentes questions sont à l'ordre du jour de nos occupations de recherche. Notre objectif final est de mesurer les effets des pratiques enseignantes sur l'engagement des élèves dans la tâche et sur la valeur qu'ils accordent aux apprentissages en éducation physique.

Nous avons proposé, après une revue de la littérature scientifique, de construire une grille de lecture nous aidant à différencier les types de contextualisation susceptibles d'avoir des effets sur les apprentissages des élèves. Dans notre recherche à visée exploratoire, nous avons tenté de retrouver et d'affiner les différentes dimensions. II reste néanmoins à questionner les perceptions des élèves quant à cette mise en contexte des savoirs pour évaluer réellement l'impact sur l'engagement et sur la valeur qu'ils attribuent aux tâches.

\section{Bibliographie}

Amigues, R. (1994). Construction des faits d'enseignement et voies de recherche en psychologie de l'éducation. Un exemple, les technologies éducatives. Texte présenté pour l'habilitation à diriger des recherches. Aix-en-Provence : Université de Provence.

Beckers, J. (2002). Développer des compétences à l'école. Vers plus d'efficacité et d'équité. Bruxelles : Labor.

Bédard, D., Frenay, M., Turgeon, J., \& Paquay, L. (2000). Les fondements de dispositifs pédagogiques visant à favoriser le transfert de connaissances: les perspectives 
de "l'apprentissage et de l'enseignement contextualisés authentiques ». Res Academica, 18 (1-2), 21-47.

Brown, J. S., Collins, A., \& Duguid, P. (1989). Situated cognition and the culture of learning. Educational Research, 18, 32-42.

Carlier, Gh. \&, Renard, J.P. (2004). Formation continue. Expertise des formateurs et identité professionnelle des formés en éducation physique. Namur : E.M.E.

Clauw, C., Dufays, J-L., Thyrion, F., Vercruysse, B., Carlier, G., Paquay, L., \& Mottier Lopez, L. (2006). Comment les enseignants du secondaire supérieur favorisent-ils un apprentissage contextualisé authentique ? Revue de la littérature et recherche exploratoire dans des classes de français et d'éducation physique. Namur: Communication au $4^{\text {ème }}$ congrès de chercheurs en éducation.

Clot, Y. (1999). La fonction psychologique du travail. Paris : PUF.

Clot, Y., \& Faïta D. (2000). Genre et style en analyse du travail. Travailler, 4, 7-42.

Collins, A., Brown, J. S., \& Holum, A. (1991). Cognitive apprenticeship : Making thinking visible. American Educator, 6-46.

Eurydice (2002). Compétences clés, un concept en développement dans l'enseignement général obligatoire. Bruxelles : Commission Européenne.

Elliot, A.J., \& Dweck, D.S. (2005). Handbook of Competence and Motivation. New York : Guilford Press.

Frenay, M., \& Bédard, D. (2004). Des dispositifs de formation universitaire s'inscrivant dans la perspective d'un apprentissage et d'un enseignement contextualisés pour favoriser la construction de connaissances et leur transfert. In A. Presseau \& M. Frenay (Eds.), le transfert des apprentissages: comprendre pour mieux intervenir (pp. 241-268). Québec : Les Presses de l'Université Laval.

Lave, J. \& Wenger, E. (1991). Situated learning : Legitimate peripheral participation. New York : Cambridge University Press.

Le Boterf, G. (1997). De la compétence à la navigation professionnelle. Paris: Les éditions d'organisation.

Mottier Lopez, L. (2003). Les structures de participation privilégiées dans une microculture de classe : un indice de l'efficacité des pratiques d'enseignement et d'apprentissage ? Dossier des sciences de l'éducation, 10, 59-75.

Mottier-Lopez, L., \& Allal, L. (2004). Participer à des pratiques d'une communauté classe : un processus de construction de significations socialement reconnues et partagées. In C. Moro \& R. Rickenmann R (eds.), Situation éducative et significations. Bruxelles : De Boeck Université. 
Mottier Lopez, L. (2005). Co-constitution de la microculture de classe dans une perspective située: Etudes d'activités de résolutions de problèmes en troisième année primaire. Thèse en sciences de l'éducation. Université de Genève.

Oddone, I., Rey, A., \& Briante, G., (1981). Redécouvrir l'expérience ouvrière. Vers une autre psychologie du travail. Paris : Ed. Sociales.

Paquay, L., Carlier, Gh., Collès, L., \& Huynen, A.-M. (éds) (2001). L'évaluation des compétences chez l'apprenant. Pratiques, méthodes et fondements. Louvain-laNeuve : Presses universitaires de Louvain.

Parmentier Ph., Paquay, L., \& coll. (2002). En quoi les situations d'enseignement apprentissage favorisent-elles la construction de compétences ? Développement d'un outil d'analyse : le Comp.A.S. Document de travail, Louvain-la Neuve.

Perrenoud, Ph. (1997). Construire des compétences dès l'école. Paris : ESF.

Pintrich, D.R., \& Shunck D. H. (1996). Motivation in education. Theory, research and applications. Englewood Cliffs, New Jersey : Prentice Hall.

Pintrich, D.R. (2003). A Motivational science perspective on the role of student motivation in learning and teaching contexts. Journal of Educational Psychology, 95 (4), 667686.

Rey, B. (2003). Les compétences à l'école : apprentissage et évaluation. Bruxelles : De Boeck.

Roegiers, X. (2000). Une pédagogie de l'intégration. Compétences et intégration des acquis dans l'enseignement. Bruxelles : De Boeck.

Romainville, M. (2001). Les implications didactiques de l'approche par compétences. Enjeux, 51, 199-223.

Sauvegrain J.-P., \& Terrisse A. (2003). Analyse de la décision d'élèves à l'épreuve de combat : une étude de cas dans un cycle de lutte en EPS, in C. Amade-Escot , Didactique de l'EPS; état des recherches (pp. 339-367). Paris Editions Revue EP.S,.

Tardif, J. \& Presseau, A. (1998). Quelques contributions de la recherche pour favoriser le transfert des apprentissages. Vie pédagogique, 108, 39-45.

Tardif, J. (1999). Le transfert des apprentissages. Montréal : Les Éditions Logiques.

Vercruysse, B. (2005). A la recherche de l'expertise d'enseignants en éducation physique: compétences, genre et styles. Mémoire de licence en éducation physique non publié. Louvain-la-Neuve : Université catholique de Louvain. 